\title{
Frame Multiresolution Analysis on Local Fields of Positive Characteristic
}

\author{
Firdous A. Shah \\ Department of Mathematics, University of Kashmir, South Campus, Anantnag, Jammu and Kashmir 192101, India
}

Correspondence should be addressed to Firdous A. Shah; fashahku@gmail.com

Received 21 August 2014; Accepted 12 January 2015

Academic Editor: Gerd Teschke

Copyright (C) 2015 Firdous A. Shah. This is an open access article distributed under the Creative Commons Attribution License, which permits unrestricted use, distribution, and reproduction in any medium, provided the original work is properly cited.

\begin{abstract}
We present a notion of frame multiresolution analysis on local fields of positive characteristic based on the theory of shift-invariant spaces. In contrast to the standard setting, the associated subspace $V_{0}$ of $L^{2}(K)$ has a frame, a collection of translates of the scaling function $\varphi$ of the form $\left\{\varphi(\cdot-u(k)): k \in \mathbb{N}_{0}\right\}$, where $\mathbb{N}_{0}$ is the set of nonnegative integers. We investigate certain properties of multiresolution subspaces which provides the quantitative criteria for the construction of frame multiresolution analysis (FMRA) on local fields of positive characteristic. Finally, we provide a characterization of wavelet frames associated with FMRA on local field $K$ of positive characteristic using the shift-invariant space theory.
\end{abstract}

\section{Introduction}

Multiresolution analysis is considered as the heart of wavelet theory. The concept of multiresolution analysis provides a natural framework for understanding and constructing discrete wavelet systems. A multiresolution analysis is an increasing family of closed subspaces $\left\{V_{j}: j \in \mathbb{Z}\right\}$ of $L^{2}(\mathbb{R})$ such that $\bigcap_{j \in \mathbb{Z}} V_{j}=\{0\}$ and $\bigcup_{j \in \mathbb{Z}} V_{j}$ is dense in $L^{2}(\mathbb{R})$ which satisfies $f \in V_{j}$ if and only if $f(2 \cdot) \in V_{j+1}$. Furthermore, there exists an element $\varphi \in V_{0}$ such that the collection of integer translates of function $\varphi,\{\varphi(\cdot-k): k \in \mathbb{Z}\}$, represents a complete orthonormal system for $V_{0}$. The function $\varphi$ is called the scaling function or the father wavelet. The concept of multiresolution analysis has been extended in various ways in recent years. These concepts are generalized to $L^{2}\left(\mathbb{R}^{d}\right)$, to lattices different from $\mathbb{Z}^{d}$, allowing the subspaces of multiresolution analysis to be generated by Riesz basis instead of orthonormal basis, admitting a finite number of scaling functions, replacing the dilation factor 2 by an integer $M \geq 2$ or by an expansive matrix $A \in G L_{d}(\mathbb{R})$ as long as $A \subset A \mathbb{Z}^{d}$ (see $[1,2])$.

On the other hand, this elegant tool for the construction of wavelet bases has been extensively studied by several authors on the various spaces, namely, Cantor dyadic groups [3], locally compact Abelian groups [4], $p$-adic fields
[5], zero-dimensional groups [6], and Vilenkin groups [7]. Recently, R. L. Benedetto and J. J. Benedetto [8] developed a wavelet theory for local fields and related groups. They did not develop the multiresolution analysis (MRA) approach; their method is based on the theory of wavelet sets. The local fields are essentially of two types: zero and positive characteristic (excluding the connected local fields $\mathbb{R}$ and $\mathbb{C}$ ). Examples of local fields of characteristic zero include the $p$-adic field $\mathbb{Q}_{p}$ whereas local fields of positive characteristic are the Cantor dyadic group and the Vilenkin $p$-groups. The structures and metrics of the local fields of zero and positive characteristic are similar, but their wavelet and MRA theory are quite different. The concept of multiresolution analysis on a local field $K$ of positive characteristic was introduced by Jiang et al. [9]. They pointed out a method for constructing orthogonal wavelets on local field $K$ with a constant generating sequence. Subsequently, tight wavelet frames on local fields of positive characteristic were constructed by Shah and Debnath [10] using extension principles. As far as the characterization of wavelets on local fields is concerned, Behera and Jahan [11] have given the characterization of all wavelets associated with multiresolution analysis on local field $K$ based on results on affine and quasiaffine frames. Recently, Shah and Abdullah [12] have introduced the notion of nonuniform multiresolution analysis on local field $K$ of positive characteristic and 
obtained the necessary and sufficient condition for a function $\varphi$ to generate a nonuniform multiresolution analysis on local fields. More results in this direction can also be found in $[13,14]$ and the references therein.

Since the use of multiresolution analysis has proven to be a very efficient tool in wavelet theory mainly because of its simplicity, it is of interest to try to generalize this notion as much as possible while preserving its connection with wavelet analysis. In this connection, Benedetto and $\mathrm{Li}$ [15] considered the dyadic semiorthogonal frame multiresolution analysis of $L^{2}(\mathbb{R})$ with a single scaling function and successfully applied the theory in the analysis of narrow band signals. The characterization of the dyadic semiorthogonal frame multiresolution analysis with a single scaling function admitting a single frame wavelet whose dyadic dilations of the integer translates form a frame for $L^{2}(\mathbb{R})$ was obtained independently by Benedetto and Treiber by a direct method [16] and by Kim and Lim by using the theory of shift-invariant spaces [17]. Later on, Yu [18] extended the results of Benedetto and Li's theory of FMRA to higher dimensions with arbitrary integral expansive matrix dilations and has established the necessary and sufficient conditions to characterize semiorthogonal multiresolution analysis frames for $L^{2}\left(\mathbb{R}^{n}\right)$.

In this paper, we introduce the notion of frame multiresolution analysis (FMRA) on local field $K$ of positive characteristic by extending the above described methods. We first investigate the properties of multiresolution subspaces, which will provide the quantitative criteria for the construction of FMRA on local fields of positive characteristic. We also show that the scaling property of an FMRA also holds for the wavelet subspaces and that the space $L^{2}(K)$ can be decomposed into the orthogonal sum of these wavelet subspaces. Finally, we study the characterization of wavelet frames associated with FMRA on local field $K$ of positive characteristic using the shift-invariant space theory.

The paper is organized as follows. In Section 2, we discuss some preliminary facts about local fields of positive characteristic including the definition of a frame. The notion of frame multiresolution analysis of $L^{2}(K)$ is introduced in Section 3 and its quantitative criteria are given by means of Theorem 12. In Section 4, we establish a complete characterization of wavelet frames generated by a finite number of mother wavelets on local field $K$ of positive characteristic.

\section{Preliminaries on Local Fields}

Let $K$ be a field and a topological space. Then, $K$ is called a local field if both $K^{+}$and $K^{*}$ are locally compact Abelian groups, where $K^{+}$and $K^{*}$ denote the additive and multiplicative groups of $K$, respectively. If $K$ is any field and is endowed with the discrete topology, then $K$ is a local field. Further, if $K$ is connected, then $K$ is either $\mathbb{R}$ or $\mathbb{C}$. If $K$ is not connected, then it is totally disconnected. Hence, by a local field, we mean a field $K$ which is locally compact, nondiscrete, and totally disconnected. The $p$-adic fields are examples of local fields. More details are referred to in $[19,20]$. In the rest of this paper, we use $\mathbb{N}, \mathbb{N}_{0}$, and $\mathbb{Z}$ to denote the sets of natural and nonnegative integers and integers, respectively.
Let $K$ be a fixed local field. Then, there is an integer $q=\mathfrak{p}^{r}$, where $\mathfrak{p}$ is a fixed prime element of $K$ and $r$ is a positive integer, and a norm $|\cdot|$ on $K$ such that for all $x \in K$ we have $|x| \geq 0$ and for each $x \in K \backslash\{0\}$ we get $|x|=q^{k}$ for some integer $k$. This norm is non-Archimedean; that is, $|x+y| \leq$ $\max \{|x|,|y|\}$ for all $x, y \in K$ and $|x+y|=\max \{|x|,|y|\}$ whenever $|x| \neq|y|$. Let $d x$ be the Haar measure on the locally compact, topological group $(K,+)$. This measure is normalized so that $\int_{\mathfrak{D}} d x=1$, where $\mathfrak{D}=\{x \in K:|x| \leq 1\}$ is the ring of integers in $K$. Define $\mathfrak{B}=\{x \in K:|x|<1\}$. The set $\mathfrak{B}$ is called the prime ideal in $K$. The prime ideal in $K$ is the unique maximal ideal in $\mathfrak{D}$, and hence as a result $\mathfrak{B}$ is both principal and prime. Therefore, for such an ideal $\mathfrak{B}$ in $\mathfrak{D}$, we have $\mathfrak{B}=\langle\mathfrak{p}\rangle=\mathfrak{p} \mathfrak{D}$.

Let $\mathfrak{D}^{*}=\mathfrak{D} \backslash \mathfrak{B}=\{x \in K:|x|=1\}$. Then, it is easy to verify that $\mathfrak{D}^{*}$ is a group of units in $K^{*}$ and if $x \neq 0$, then we may write $x=\mathfrak{p}^{k} x^{\prime}, x^{\prime} \in \mathfrak{D}^{*}$. Moreover, each $\mathfrak{B}^{k}=\mathfrak{p}^{k} \mathfrak{D}=$ $\left\{x \in K:|x|<q^{-k}\right\}$ is a compact subgroup of $K^{+}$and is known as the fractional ideals of $K^{+}$(see [19]). Let $\mathcal{U}=\left\{a_{i}\right\}_{i=0}^{q-1}$ be any fixed full set of coset representatives of $\mathfrak{B}$ in $\mathfrak{D}$; then, every element $x \in K$ can be expressed uniquely as $x=\sum_{\ell=k}^{\infty} c_{\ell} \mathfrak{p}^{\ell}$ with $c_{\ell} \in \mathcal{U}$. Let $\chi$ be a fixed character on $K^{+}$that is trivial on $\mathfrak{D}$ but is nontrivial on $\mathfrak{B}^{-1}$. Therefore, $\chi$ is constant on cosets of $\mathfrak{D}$, implying that if $y \in \mathfrak{B}^{k}$, then $\chi_{y}(x)=\chi(y x)$ for $x \in$ $K$. Suppose that $\chi_{u}$ is any character on $K^{+}$; then, clearly the restriction $\chi_{\mathfrak{u}} \mid \mathfrak{D}$ is also a character on $\mathfrak{D}$. Therefore, if $\{u(n)$ : $\left.n \in \mathbb{N}_{0}\right\}$ is a complete list of distinct coset representatives of D in $K^{+}$, then, as it was proved in [20], the set $\left\{\chi_{u(n)}: n \in \mathbb{N}_{0}\right\}$ of distinct characters on $\mathfrak{D}$ is a complete orthonormal system on $\mathfrak{D}$.

The Fourier transform $\widehat{f}$ of a function $f \in L^{1}(K) \cap L^{2}(K)$ is defined by

$$
\widehat{f}(\xi)=\int_{K} f(x) \overline{\chi_{\xi}(x)} d x .
$$

It is noted that

$$
\widehat{f}(\xi)=\int_{K} f(x) \overline{\chi_{\xi}(x)} d x=\int_{K} f(x) \chi(-\xi x) d x .
$$

Furthermore, the properties of Fourier transform on local field are much similar to those on the real line. In particular, Fourier transform is unitary on $L^{2}(K)$.

We now impose a natural order on the sequence $\{u(n)$ : $\left.n \in \mathbb{N}_{0}\right\}$. Since $\mathfrak{D} / \mathfrak{B} \cong G F(q)$, where $G F(q)$ is a $c$-dimensional vector space over the field $G F(q)$ (see [20]), we choose a set $\left\{1=\zeta_{0}, \zeta_{1}, \zeta_{2}, \ldots, \zeta_{c-1}\right\} \subset \mathfrak{D}^{*}$ such that $\operatorname{span}\left\{\zeta_{j}\right\}_{j=0}^{c-1} \cong G F(q)$. For $n \in \mathbb{N}_{0}$ such that $0 \leq n<q$, we have

$$
\begin{array}{r}
n=a_{0}+a_{1} p+\cdots+a_{c-1} p^{c-1}, \quad 0 \leq a_{k}<p, \\
k=0,1, \ldots, c-1 .
\end{array}
$$

Define

$$
u(n)=\left(a_{0}+a_{1} \zeta_{1}+\cdots+a_{c-1} \zeta_{c-1}\right) \mathfrak{p}^{-1} .
$$

For $n \in \mathbb{N}_{0}$ and $0 \leq b_{k}<q, k=0,1,2, \ldots, s$, we write

$$
n=b_{0}+b_{1} q+b_{2} q^{2}+\cdots+b_{s} q^{s}
$$


such that

$$
u(n)=u\left(b_{0}\right)+u\left(b_{1}\right) \mathfrak{p}^{-1}+\cdots+u\left(b_{s}\right) \mathfrak{p}^{-s} .
$$

Also, for $r, k \in \mathbb{N}_{0}$ and $0 \leq s<q^{k}$, we have

$$
u\left(r q^{k}+s\right)=u(r) \mathfrak{p}^{-k}+u(s) .
$$

Further, it is easy to verify that $u(n)=0$ if and only if $n=0$ and $\left\{u(\ell)+u(k): k \in \mathbb{N}_{0}\right\}=\left\{u(k): k \in \mathbb{N}_{0}\right\}$ for a fixed $\ell \in \mathbb{N}_{0}$. Hereafter, we use the notation $\chi_{n}:=\chi_{u(n)}, n \geq 0$.

Let the local field $K$ be of characteristic $p>0$ and let $\zeta_{0}, \zeta_{1}, \zeta_{2}, \ldots, \zeta_{c-1}$ be as above. We define a character $\chi$ on $K$ as follows:

$$
\chi\left(\zeta_{\mu} \mathfrak{p}^{-j}\right)= \begin{cases}\exp \left(\frac{2 \pi i}{p}\right), & \mu=0, j=1, \\ 1, & \mu=1, \ldots, c-1 \text { or } j \neq 1 .\end{cases}
$$

Definition 1. Let $\mathbb{Q}$ be a separable Hilbert space. A sequence $\left\{f_{k}: k \in \mathbb{N}_{0}\right\}$ in $\mathbb{W}$ is called a frame for $\mathbb{U}$ if there exist constants $A$ and $B$ with $0<A \leq B<\infty$ such that

$$
A\|f\|_{2}^{2} \leq \sum_{k \in \mathbb{N}_{0}}\left|\left\langle f, f_{k}\right\rangle\right|^{2} \leq B\|f\|_{2}^{2}, \quad \forall f \in \mathbb{H} .
$$

The largest constant $A$ and the smallest constant $B$ satisfying (9) are called the upper and the lower frame bound, respectively. A frame is said to be tight if it is possible to choose $A=B$ and a frame is said to be exact if it ceases to be a frame when any one of its elements is removed. An exact frame is also known as a Riesz basis.

The following theorem gives us an elementary characterization of frames.

Theorem 2 (see [15]). A sequence $\left\{f_{k}: k \in \mathbb{N}_{0}\right\}$ in a Hilbert space $\mathbb{U}$ is a frame for $\mathbb{U}$ if and only if there exists a sequence $a=\left\{a_{k}\right\} \in l^{2}\left(\mathbb{N}_{0}\right)$ with $\|a\|_{l^{2}\left(\mathbb{N}_{0}\right)} \leq C\|f\|, C>0$, such that

$$
f=\sum_{k \in \mathbb{N}_{0}} a_{k} f_{k}
$$

and $\sum_{k \in \mathbb{N}_{0}}\left|\left\langle f, f_{k}\right\rangle\right|^{2}<\infty$, for every $f \in \mathbb{H}$.

For $j \in \mathbb{Z}$ and $y \in K$, we define the dilation operator $\delta_{j}$ and the translation operator $\tau_{y}$ as follows:

$$
\begin{array}{r}
\delta_{j} f(x)=q^{j / 2} f\left(\mathfrak{p}^{-j} x\right), \quad \tau_{y} f(x)= \\
f(x-y), \\
f \in L^{2}(K) .
\end{array}
$$

Our study uses the theory of shift-invariant spaces developed in $[21,22]$ and the references therein. A closed subspace $S$ of $L^{2}(K)$ is said to be shift-invariant if $\tau_{k} f \in S$ whenever $f \in S$ and $k \in \mathbb{N}_{0}$. A closed shift-invariant subspace $S$ of $L^{2}(K)$ is said to be generated by $\Phi \subset L^{2}(K)$ if $S=\overline{\operatorname{span}}\left\{\tau_{k} \varphi(x):=\right.$ $\left.\varphi(x-u(k)): k \in \mathbb{N}_{0}, \varphi \in \Phi\right\}$. The cardinality of the smallest generating set $\Phi$ for $S$ is called the length of $S$ which is denoted by $|S|$. If $|S|=$ finite, then $S$ is called a finite shiftinvariant space (FSI) and if $|S|=1$, then $S$ is called a principal shift-invariant space (PSI). Moreover, the spectrum of a shiftinvariant space is defined to be

$$
\sigma(S)=\{\xi \in \mathfrak{D}: \widehat{S}(\xi) \neq\{0\}\},
$$

where $\widehat{S}(\xi)=\left\{\widehat{f}(\xi+u(k)) \in l^{2}\left(\mathbb{N}_{0}\right): f \in S, k \in \mathbb{N}_{0}\right\}$.

\section{Frame Multiresolution Analysis on Local Fields}

We first introduce the notion of a frame multiresolution analysis (FMRA) of $L^{2}(K)$.

Definition 3. Let $K$ be a local field of positive characteristic $p>0$ and let $\mathfrak{p}$ be a prime element of $K$. A frame multiresolution analysis of $L^{2}(K)$ is a sequence of closed subspaces $\left\{V_{j}: j \in \mathbb{Z}\right\}$ of $L^{2}(K)$ satisfying the following properties:

(a) $V_{j} \subset V_{j+1}$ for all $j \in \mathbb{Z}$;

(b) $\bigcup_{j \in \mathbb{Z}} V_{j}$ is dense in $L^{2}(K)$ and $\bigcap_{j \in \mathbb{Z}} V_{j}=\{0\}$;

(c) $f(\cdot) \in V_{j}$ if and only if $f\left(\mathfrak{p}^{-1} \cdot\right) \in V_{j+1}$ for all $j \in \mathbb{Z}$;

(d) the function $f$ lying in $V_{0}$ implies that the collection $f(\cdot-u(k)) \in V_{0}$, for all $k \in \mathbb{N}_{0}$;

(e) the sequence $\left\{\tau_{k} \varphi:=\varphi(\cdot-u(k)): k \in \mathbb{N}_{0}\right\}$ is a frame for the subspace $V_{0}$.

The function $\varphi$ is known as the scaling function while the subspaces $V_{j}$ 's are known as approximation spaces or multiresolution subspaces. A frame multiresolution analysis is said to be nonexact and, respectively, exact if the frame for the subspace $V_{0}$ is nonexact and, respectively, exact. In multiresolution analysis studied in [9], the frame condition is replaced by that of an orthonormal basis or an exact frame.

Next, we establish several properties of multiresolution subspaces that will help in the construction of frame multiresolution analysis on local field $K$ of positive characteristic. The following proposition shows that, for every $j \in \mathbb{Z}$, the sequence $\left\{\varphi_{j, k}: k \in \mathbb{N}_{0}\right\}$, where

$$
\varphi_{j, k}(x)=q^{j / 2} \varphi\left(\mathfrak{p}^{-j} x-u(k)\right),
$$

is a frame for $V_{j}$.

Proposition 4. Let $\left\{\tau_{k} \varphi: k \in \mathbb{N}_{0}\right\}$ be a frame for $V_{0}=$ $\overline{\operatorname{span}}\left\{\tau_{k} \varphi: k \in \mathbb{N}_{0}\right\}$ and

$$
V_{j}=\left\{f \in L^{2}(K): f\left(\mathfrak{p}^{j} \cdot\right) \in V_{0}\right\}, \quad j \in \mathbb{Z} .
$$

Then, the sequence $\left\{\varphi_{j, k}: k \in \mathbb{N}_{0}\right\}$ defined in (13) is a frame for $V_{j}$ with the same bounds as those for $V_{0}$. 
Proof. For any $f \in V_{j}$, we have

$$
\begin{aligned}
\sum_{k \in \mathbb{N}_{0}}\left|\left\langle\delta^{-j} f, \tau_{k} \varphi\right\rangle\right|^{2} & =\sum_{k \in \mathbb{N}_{0}}\left|\int_{K} q^{-j / 2} f\left(\mathfrak{p}^{j} x\right) \overline{\varphi(x-u(k))} d x\right|^{2} \\
& =\sum_{k \in \mathbb{N}_{0}}\left|\int_{K} f(x) q^{j / 2} \overline{\varphi\left(\mathfrak{p}^{-j} x-u(k)\right)} d x\right|^{2} \\
& =\sum_{k \in \mathbb{N}_{0}}\left|\left\langle f, \varphi_{j, k}\right\rangle\right|^{2}
\end{aligned}
$$

Since $\left\{\tau_{k} \varphi: k \in \mathbb{N}_{0}\right\}$ is a frame for $V_{0}$, therefore we have

$$
\begin{aligned}
A\|f\|_{2}^{2} & =A\left\|\delta^{-j} f\right\|_{2}^{2} \leq \sum_{k \in \mathbb{N}_{0}}\left|\left\langle f, \varphi_{j, k}\right\rangle\right|^{2} \leq B\left\|\delta^{-j} f\right\|_{2}^{2} \\
& =B\|f\|_{2}^{2} .
\end{aligned}
$$

This completes the proof of the proposition.

We now characterize all functions of FSI space by virtue of its Fourier transforms.

Proposition 5. Let $\left\{\tau_{k} \varphi: k \in \mathbb{N}_{0}, \varphi \in \Phi\right\}$ be a frame for its closed linear span $V$, where $\Phi=\left\{\varphi_{1}, \varphi_{2}, \ldots, \varphi_{L}\right\} \subset L^{2}(K)$. Then, $f \in L^{2}(K)$ lies in $V$ if and only if there exist integral periodic functions $h_{\ell} \in L^{2}(\mathfrak{D}), \ell=1, \ldots, L$, such that

$$
\widehat{f}(\xi)=\sum_{\ell=1}^{L} h_{\ell}(\xi) \widehat{\varphi}_{\ell}(\xi) .
$$

Proof. Since the system $\left\{\tau_{k} \varphi: k \in \mathbb{N}_{0}, \varphi \in \Phi\right\}$ is a frame for $V$, then, by Theorem 2 , there exists a sequence $\left\{a_{k}^{\ell}\right\} \in l^{2}\left(\mathbb{N}_{0}\right)$, for $\ell=1, \ldots, L$, such that

$$
f(x)=\sum_{\ell=1}^{L} \sum_{k \in \mathbb{N}_{0}} a_{k}^{\ell} \varphi_{\ell}(x-u(k)) .
$$

Taking Fourier transform on both sides of (20), we obtain

$$
\widehat{f}(\xi)=\sum_{\ell=1}^{L} h_{\ell}(\xi) \widehat{\varphi}_{\ell}(\xi),
$$

where $h_{\ell}(\xi)=\sum_{k \in \mathbb{N}_{0}} a_{k}^{\ell} \chi_{k}(\xi)$ are the integral periodic functions in $L^{2}(\mathfrak{D})$. The converse is established by taking $h_{\ell}$ as above and applying the inverse Fourier transform on both sides of (19).

We now study some properties of the multiresolution subspaces $V_{j}$ of the form (14) by means of the Fourier transform.

Proposition 6. Let $\left\{\tau_{k} \varphi: k \in \mathbb{N}_{0}\right\}$ be a frame for $V_{0}=$ $\overline{\operatorname{span}}\left\{\tau_{k} \varphi: k \in \mathbb{N}_{0}\right\}$ and, for $j \in \mathbb{Z}$, define $V_{j}$ by (14). Then, for any function $\psi \in V_{1}$, there exists periodic function $G \in L^{2}(\mathfrak{D})$ such that

$$
\widehat{\psi}\left(\mathfrak{p}^{-1} \xi\right)=q^{1 / 2} G(\xi) \widehat{\varphi}(\xi)
$$

Proof. By the definition of $V_{j}$, it follows that $\psi(\mathfrak{p} \cdot) \in V_{0}$. By Proposition 5, there exists a periodic function $G \in L^{2}(\mathfrak{D})$ such that $(\psi(\mathfrak{p} \cdot))^{\wedge}=\widehat{\psi}\left(\mathfrak{p}^{-1} \xi\right)=q^{1 / 2} G(\xi) \widehat{\varphi}(\xi)$ lies in $L^{2}(K)$.

The following theorem establishes a sufficient condition to ensure that the nesting property holds for the subspaces $V_{j}$ 's.

Theorem 7. Let $\left\{\tau_{k} \varphi: k \in \mathbb{N}_{0}\right\}$ be a frame for $V_{0}=$ $\overline{\operatorname{span}}\left\{\tau_{k} \varphi: k \in \mathbb{N}_{0}\right\}$ and, for $j \in \mathbb{Z}$, define $V_{j}$ by (14). Assume that there exists a periodic function $H \in L^{\infty}(\mathfrak{D})$ such that

$$
\widehat{\varphi}(\xi)=q^{1 / 2} H(\mathfrak{p} \xi) \widehat{\varphi}(\mathfrak{p} \xi) .
$$

Then, $V_{j} \subseteq V_{j+1}$, for every $j \in \mathbb{Z}$.

Proof. Given any $f \in V_{j}$, there exists a sequence $\left\{a_{k}\right\}_{k \in \mathbb{N}_{0}} \in$ $l^{2}\left(\mathbb{N}_{0}\right)$ such that

$$
f(x)=q^{j / 2} \sum_{k \in \mathbb{N}_{0}} a_{k} \varphi\left(\mathfrak{p}^{-j} x-u(k)\right) .
$$

Let $m_{0}(\xi)=\sum_{k \in \mathbb{N}_{0}} a_{k} \chi_{k}(\xi) \in L^{2}(\mathfrak{D})$ and let $m_{1}(\mathfrak{p} \xi)=$ $m_{0}(\xi) H(\mathfrak{p} \xi)$. Then, clearly $m_{1}$ lies in $L^{2}(\mathfrak{D})$ as $H$ lies in $L^{\infty}(\mathfrak{D})$. Therefore, by Parseval's identity, there exists a sequence $\left\{b_{k}\right\}_{k \in \mathbb{N}_{0}} \in l^{2}\left(\mathbb{N}_{0}\right)$ such that $m_{1}(\xi)=\sum_{k \in \mathbb{N}_{0}} b_{k} \chi_{k}(\xi)$ lies in $L^{2}(K)$.

Taking Fourier transform of (24) and using assumption (23), we obtain

$$
\begin{aligned}
\widehat{f}(\xi) & =q^{j / 2} m_{0}\left(\mathfrak{p}^{j} \xi\right) \widehat{\varphi}\left(\mathfrak{p}^{j} \xi\right) \\
& =q^{(j+1) / 2} m_{0}\left(\mathfrak{p}^{j} \xi\right) H\left(\mathfrak{p}^{j+1} \xi\right) \widehat{\varphi}\left(\mathfrak{p}^{j+1} \xi\right) \\
& =q^{(j+1) / 2} m_{1}\left(\mathfrak{p}^{j+1} \xi\right) \widehat{\varphi}\left(\mathfrak{p}^{j+1} \xi\right) .
\end{aligned}
$$

By implementing inverse Fourier transform to (25), we have

$$
f(x)=q^{(j+1) / 2} \sum_{k \in \mathbb{N}_{0}} b_{k} \varphi\left(\mathfrak{p}^{-j-1} x-u(k)\right) .
$$

Using Proposition 4, we observe that $f \in V_{j+1}$. Moreover, it is easy to verify that the function $H$ in (23) is not unique.

The following theorem is the converse to Theorem 7.

Theorem 8. Let $\left\{\tau_{k} \varphi: k \in \mathbb{N}_{0}\right\}$ be a frame for $V_{0}=$ $\overline{\operatorname{span}}\left\{\tau_{k} \varphi: k \in \mathbb{N}_{0}\right\}$ and, for $j \in \mathbb{Z}$, define $V_{j}$ by (14). Assume that $V_{0} \subseteq V_{1}$ and $\Phi(\xi)=\|\widehat{\varphi}(\xi-u(k))\|_{l^{2}\left(\mathbb{N}_{0}\right)}^{2}$. Then, there exists periodic function $H \in L^{\infty}(\mathfrak{D})$ such that (23) holds.

Proof. Since $\left\{\tau_{k} \varphi: k \in \mathbb{N}_{0}\right\}$ is a frame for $V_{0}$, therefore there exist positive constants $A$ and $B$ such that

$$
A \leq \Phi(\xi) \leq B \quad \text { a.e. on } \sigma\left(V_{0}\right) .
$$

Since $V_{0} \subseteq V_{1}$, we have $\varphi \in V_{1}$. By Proposition 6, there exists a periodic function $H_{0} \in L^{2}$ (D) such that

$$
\widehat{\varphi}\left(\mathfrak{p}^{-1} \xi\right)=q^{1 / 2} H_{0}(\xi) \widehat{\varphi}(\xi)
$$


Therefore, we have

$$
|\widehat{\varphi}(\xi)|^{2}=q\left|H_{0}(\mathfrak{p} \xi)\right|^{2}|\widehat{\varphi}(\mathfrak{p} \xi)|^{2} \quad \text { a.e. }
$$

Let $\mathcal{S}=\mathfrak{B} \backslash \sigma\left(V_{0}\right)$ and $H \in L^{2}(\mathfrak{D})$ be a periodic function such that $H=H_{0}$, a.e. on $\sigma\left(V_{0}\right)$, and $H$ is bounded on $\mathcal{S}$ by a positive constant $C$. Then, it follows from the above fact that $H$ is not unique so that (29) also holds for $H$; that is,

$$
|\widehat{\varphi}(\xi)|^{2}=q|H(\mathfrak{p} \xi)|^{2}|\widehat{\varphi}(\mathfrak{p} \xi)|^{2} \quad \text { a.e. }
$$

Taking $n=k p+r$, where $k \in \mathbb{N}_{0}$ and $r=0,1, \ldots, q-1$, we have

$$
\begin{aligned}
& |\widehat{\varphi}(\xi+u(n))|^{2} \\
& \quad=q|H(\mathfrak{p} \xi+\mathfrak{p} u(r))|^{2} \mid \widehat{\varphi}\left(\mathfrak{p} \xi+\mathfrak{p} u(r)+\left.u(k)\right|^{2} \quad\right. \text { a.e. }
\end{aligned}
$$

Summing up (31) for all $k \in \mathbb{N}_{0}$ and $r=0,1, \ldots, q-1$, we have

$$
\begin{aligned}
& \sum_{n \in \mathbb{N}_{0}}|\widehat{\varphi}(\xi+u(n))|^{2} \\
& \quad=q \sum_{r=0}^{q-1}|H(\mathfrak{p} \xi+\mathfrak{p} u(r))|^{2} \sum_{k \in \mathbb{N}_{0}} \mid \widehat{\varphi}\left(\mathfrak{p} \xi+\mathfrak{p} u(r)+\left.u(k)\right|^{2} \quad\right. \text { a.e., }
\end{aligned}
$$

which is equivalent to

$$
\Phi(\xi)=q \sum_{r=0}^{q-1}|H(\mathfrak{p} \xi+\mathfrak{p} u(r))|^{2} \Phi(\mathfrak{p} \xi+\mathfrak{p} u(r)) \quad \text { a.e. }
$$

or

$$
\Phi\left(\mathfrak{p}^{-1} \xi\right)=q \sum_{r=0}^{q-1}|H(\xi+\mathfrak{p} u(r))|^{2} \Phi(\xi+\mathfrak{p} u(r)) \quad \text { a.e. }
$$

Note that $\Phi\left(\mathfrak{p}^{-1} \xi\right) \leq B$ a.e. and, hence, (34) becomes

$$
\sum_{r=0}^{q-1}|H(\xi+\mathfrak{p} u(r))|^{2} \Phi(\xi+\mathfrak{p} u(r)) \leq q B \quad \text { a.e. }
$$

This implies that, for almost every $\xi \in \mathfrak{B}^{-1}$ and $r=0,1, \ldots$, $q-1$, we have

$$
|H(\xi+\mathfrak{p} u(r))|^{2} \Phi(\xi+\mathfrak{p} u(r)) \leq q B .
$$

Also, if $\Phi(\xi+\mathfrak{p} u(r))=0$, then $|H(\xi+\mathfrak{p} u(r))| \leq C$ and if $\Phi(\xi+\mathfrak{p} u(r))>0$, then we may assume that $A \leq \Phi(\xi+\mathfrak{p} u(r)) \leq$ $B$. Thus, for almost every $\xi \in \mathfrak{B}^{-1}$ and $r=0,1, \ldots, q-1$, we have

$$
|H(\xi+\mathfrak{p} u(r))|^{2} \leq \max \left\{C^{2}, q B A^{-1}\right\} .
$$

Hence, $H$ is essentially bounded on $\mathfrak{D}$. This proves the theorem completely.

The following two propositions are proved in [23].
Proposition 9. Suppose $V_{0}=\overline{\operatorname{span}}\left\{\tau_{k} \varphi: k \in \mathbb{N}_{0}\right\}$ and, for each $j \in \mathbb{Z}$, define $V_{j}$ by (14) such that $V_{0} \subseteq V_{1}$. Assume that $|\widehat{\varphi}|>0$, a.e on a neighborhood of zero. Then, the union $\bigcup_{j \in \mathbb{Z}} V_{j}$ is dense in $L^{2}(K)$.

Proposition 10. Let $\varphi \in L^{2}(K)$ and define $V_{0}=\overline{\operatorname{span}}\left\{\tau_{k} \varphi\right.$ : $\left.k \in \mathbb{N}_{0}\right\}$. For each $j \in \mathbb{Z}$, define $V_{j}$ by (14). Then, one has $\bigcap_{j \in \mathbb{Z}} V_{j}=\{0\}$.

Lemma 11. Let $V_{j}$ be the family of subspaces defined by (14) with $V_{j} \subseteq V_{j+1}$, for each $j \in \mathbb{Z}$. Suppose $\varphi \in L^{2}(K)$ is a nonzero function with $V_{0}=\overline{\operatorname{span}}\left\{\tau_{k} \varphi: k \in \mathbb{N}_{0}\right\}$. Then, for every $j \in$ $\mathbb{Z}, V_{j}$ is a proper subspace of $V_{j+1}$.

Proof. Suppose that $V_{\ell}=V_{\ell+1}$ for some $\ell \in \mathbb{Z}$. Let $f \in V_{j+1}$; then, for any given $j \in \mathbb{Z}$, we have $f\left(\mathfrak{p}^{j+1-\ell-1} x\right) \in V_{j+1}$. Since $f\left(\mathfrak{p}^{j-\ell} x\right) \in V_{\ell}$, therefore $f$ lies in $V_{j}$ and $V_{j}=V_{j+1}$. Hence, $\bigcap_{j \in \mathbb{Z}} V_{j}=V_{0}$. By Proposition 10, it follows that $V_{j}=\{0\}$, which is a contradiction.

Combining all our results so far, we have the following theorem.

Theorem 12. Let $\varphi \in L^{2}(K)$ and define $V_{0}=\overline{\operatorname{span}}\left\{\tau_{k} \varphi: k \in\right.$ $\left.\mathbb{N}_{0}\right\}$. For each $j \in \mathbb{Z}$, define $V_{j}$ by (14) and $\Phi(\xi)=\| \widehat{\varphi}(\xi-$ $u(k)) \|_{l^{2}\left(\mathbb{N}_{0}\right)}^{2}$. Suppose that the following hold:

(i) $A \leq \Phi(\xi) \leq B$ a.e. on $\sigma\left(V_{0}\right)$,

(ii) there exists a periodic function $H \in L^{\infty}$ (D) such that

$$
\widehat{\varphi}(\xi)=q^{1 / 2} H(\mathfrak{p} \xi) \widehat{\varphi}(\mathfrak{p} \xi), \quad \text { a.e. }
$$

(iii) $|\widehat{\varphi}|>0$, a.e. on a neighborhood of zero.

Then, $\left\{V_{j}: j \in \mathbb{Z}\right\}$ defines a frame multiresolution analysis of $L^{2}(K)$.

Proof. Since $V_{0}$ is a shift-invariant subspace of $L^{2}(K)$, therefore the system $\left\{\tau_{k} \varphi: k \in \mathbb{N}_{0}\right\}$ forms a frame for $V_{0}$ with frame bounds $A$ and $B$. By Theorem 7 and Lemma 11, it follows that $V_{j} \subset V_{j+1}$, for every $j \in \mathbb{Z}$. Hence, by the definition of $V_{j}, f$ lies in $V_{j}$ if and only if $f\left(\mathfrak{p}^{j}\right.$.) lies in $V_{0}$, while $f\left(\mathfrak{p}^{-1} \cdot\right)$ lies in $V_{j+1}$ if and only if $f\left(\mathfrak{p}^{j+1} \cdot\right)$ lies in $V_{0}$. Thus, $f$ lies in $V_{j}$ if and only if $f\left(\mathfrak{p}^{-1} \cdot\right)$ lies in $V_{j+1}$. Moreover, by assumption (iii) and Proposition 10, it follows that $\bigcup_{j \in \mathbb{Z}} V_{j}$ is dense in $L^{2}(K)$ and $\bigcap_{j \in \mathbb{Z}} V_{j}=\{0\}$. Thus, the sequence $\left\{V_{j}: j \in \mathbb{Z}\right\}$ satisfies all the conditions to be a frame multiresolution analysis of $L^{2}(K)$.

In order to construct wavelet frames associated with frame multiresolution analysis on local fields $K$ of positive characteristic, we introduce the orthogonal complement subspaces $\left\{W_{j}: j \in \mathbb{Z}\right\}$ of $V_{j}$ in $V_{j+1}$. It is easy to verify that the sequence of subspaces $\left\{W_{j}: j \in \mathbb{Z}\right\}$ also satisfies the scaling property; that is,

$$
W_{j}=\left\{f \in L^{2}(K): f\left(\mathfrak{p}^{j} \cdot\right) \in W_{0}\right\}, \quad j \in \mathbb{Z} .
$$


Theorem 13. Let $\left\{V_{j}: j \in \mathbb{Z}\right\}$ be an increasing sequence of closed subspaces of $L^{2}(K)$ such that $\bigcup_{j \in \mathbb{Z}} V_{j}$ is dense in $L^{2}(K)$ and $\bigcap_{j \in \mathbb{Z}} V_{j}=\{0\}$. Let $W_{j}$ be the orthogonal complement of $V_{j}$ in $V_{j+1}$, for each $j \in \mathbb{Z}$. Then, the subspaces $W_{j}$ are pairwise orthogonal and

$$
L^{2}(K)=\bigoplus_{j \in \mathbb{Z}} W_{j}
$$

Proof. Assume that $i<j$; then, $\left\langle f_{i}, f_{j}\right\rangle=0$, for any $f_{i} \in W_{j}$ as $W_{i} \subset V_{i+1} \subset V_{j}$. Let $P_{j}$ be the orthogonal projection operators from $L^{2}(K)$ onto $V_{j}$; then, $\lim _{j \rightarrow \infty} P_{j} f=f, \lim _{j \rightarrow-\infty} P_{j} f=$ 0 , and $W_{j}=\left\{f-P_{j} f: f \in V_{j+1}\right\}$. Therefore, for any $f \in$ $L^{2}(K)$, we have

$$
f=\sum_{j \in \mathbb{Z}}\left(P_{j+1} f-P_{j} f\right) .
$$

Thus, the result of the direct sum follows since $P_{j+1}-P_{j}$ is the orthogonal projector from $L^{2}(K)$ onto $W_{j}$.

\section{Characterization of Wavelet Frames} on Local Fields

In this section, we give the characterization of wavelet frames associated with frame multiresolution analysis on local fields of positive characteristic. First, we will characterize the existence of a function $\psi$ in $W_{0}$, where $W_{0}$ is the orthogonal complement of $V_{0}$ in $V_{1}$, by virtue of the analysis filters $G$ and $H$, defined as in Section 3.

Theorem 14. Let $H$ be a periodic function associated with the frame multiresolution analysis $\left\{V_{j}: j \in \mathbb{Z}\right\}$ satisfying the condition (23). Define $W_{0}$ as the orthogonal complement of $V_{0}$ in $V_{1}$. Let $\psi \in V_{1}$ such that

$$
\widehat{\psi}(\xi)=q^{1 / 2} G(\mathfrak{p} \xi) \widehat{\varphi}(\mathfrak{p} \xi),
$$

where $G$ is a periodic function in $L^{2}(\mathfrak{D})$. Then, $\psi$ lies in $W_{0}$ if and only if

$$
\sum_{r=0}^{q-1} H(\mathfrak{p} \xi+\mathfrak{p} u(r)) \Phi(\mathfrak{p} \xi+\mathfrak{p} u(r)) \overline{G(\mathfrak{p} \xi+\mathfrak{p} u(r))}=0 \quad \text { a.e. } \xi .
$$

Proof. We note that $\psi$ lies in $W_{0}$ if and only if

$$
\left\langle\psi, \tau_{k} \psi\right\rangle=\langle\psi, \psi(\cdot-u(k))\rangle=0, \quad \forall k \in \mathbb{N}_{0} .
$$

Define

$$
F(\xi)=\sum_{k \in \mathbb{N}_{0}} \widehat{\varphi}(\xi+u(k)) \overline{\widehat{\psi}(\xi+u(k))} .
$$

Then, it is easy to verify that $F$ lies in $L^{1}(\mathfrak{D})$ by using Monotonic Convergence Theorem and the Plancherel Theorem as

$$
\begin{aligned}
\int_{\mathfrak{D}}|F(\xi)| d \xi & \leq \int_{\mathfrak{D}} \sum_{k \in \mathbb{N}_{0}}|\widehat{\varphi}(\xi+u(k)) \hat{\psi}(\xi+u(k))| d \xi \\
& =\sum_{k \in \mathbb{N}_{0}} \int_{\mathscr{D}}|\widehat{\varphi}(\xi+u(k)) \widehat{\psi}(\xi+u(k))| d \xi \\
& =\int_{K}|\widehat{\varphi}(\xi) \widehat{\psi}(\xi)| d \xi \\
& \leq\|\widehat{\varphi}\|_{2}\|\hat{\psi}\|_{2}=\|\varphi\|_{2}\|\psi\|_{2} .
\end{aligned}
$$

For a fixed $n \in \mathbb{N}_{0}$, we define $F_{M}$ as

$$
F_{M}(\xi)=\sum_{k=0}^{M} \widehat{\varphi}(\xi+u(k)) \overline{\widehat{\psi}(\xi+u(k))} \chi_{n}(\xi) .
$$

Then, in view of (23) and (42), we have

$$
F_{M}(\xi)=q \sum_{r=0}^{q-1} \sum_{q k+r \leq M} H(\mathfrak{p} \xi+\mathfrak{p} u(r))|\widehat{\varphi}(\mathfrak{p} \xi+\mathfrak{p} u(r)+u(k))|^{2}
$$

$$
\cdot \overline{G(\mathfrak{p} \xi+\mathfrak{p} u(r))} \chi_{n}(\xi) .
$$

Using Monotonic Convergence Theorem and the CauchySchwartz inequality, we obtain

$$
\begin{aligned}
& \left\|F_{M}-F \chi_{n}\right\|_{L^{2}(\mathfrak{D})} \\
& \quad \leq \int_{\mathfrak{D}} \sum_{k \geq M+1}|\widehat{\varphi}(\xi+u(k)) \widehat{\psi}(\xi+u(k))| d \xi \\
& \quad=\sum_{k \geq M+1} \int_{\mathfrak{D}}|\widehat{\varphi}(\xi+u(k)) \widehat{\psi}(\xi+u(k))| d \xi \\
& \quad=\sum_{k \geq M+1} \int_{x+\mathfrak{D}}|\widehat{\varphi}(\xi) \widehat{\psi}(\xi)| d \xi \\
& \quad \leq \int_{|\xi|>M}|\widehat{\varphi}(\xi) \widehat{\psi}(\xi)| d \xi \\
& \quad \leq\left\{\int_{|\xi|>M}|\widehat{\varphi}(\xi)|^{2} d \xi\right\}^{1 / 2}\left\{\int_{|\xi|>M}|\widehat{\psi}(\xi)|^{2} d \xi\right\}^{1 / 2} \\
& \quad \longrightarrow 0 \quad \text { as } M \longrightarrow \infty .
\end{aligned}
$$

Hence,

$$
\lim _{M \rightarrow \infty}\left\|F_{M}-F \chi_{n}\right\|_{L^{2}(\mathfrak{D})}=0 .
$$

Therefore, there exists a subsequence $\left\{F_{M_{j}}\right\}$ such that

$$
\lim _{j \rightarrow \infty}\left\|F_{M_{j}}-F \chi_{n}\right\|_{L^{2}(\mathfrak{D})}=0, \quad \text { a.e. }
$$

Hence,

$$
\begin{aligned}
F(\xi)=q \sum_{r=0}^{q-1} H(\mathfrak{p} \xi+\mathfrak{p} u(r)) \\
\cdot \Phi(\mathfrak{p} \xi+\mathfrak{p} u(r)) \overline{G(\mathfrak{p} \xi+\mathfrak{p} u(r))} \text { a.e. }
\end{aligned}
$$


Using (50) and the Dominated Convergence Theorem, we have, for all $n \in \mathbb{N}_{0}$,

$$
\begin{aligned}
\left\langle\psi, \tau_{-n} \varphi\right\rangle & =\int_{K} \widehat{\psi}(\xi) \overline{\hat{\varphi}(\xi)} \chi_{n}(\xi) d \xi \\
& =\sum_{k \in \mathbb{N}_{0}} \int_{x+\mathfrak{D}} \widehat{\psi}(\xi) \overline{\widehat{\varphi}(\xi)} \chi_{n}(\xi) d \xi \\
& =\lim _{M \rightarrow \infty} \sum_{k=0}^{M} \int_{\mathscr{D}} \widehat{\psi}(\xi+u(k)) \\
& \cdot \overline{\widehat{\varphi}(\xi+u(k))} \chi_{n}(\xi) \chi_{k}(\xi) d \xi \\
& =\lim _{M \rightarrow \infty} \int_{\mathscr{D}} F_{M}(\xi) d \xi \\
& =\int_{\mathscr{D}} F(\xi) \chi_{n}(\xi) d \xi .
\end{aligned}
$$

Consequently, $F=0$, a.e., is the necessary and sufficient condition for (44) to hold for all $n \in \mathbb{N}_{0}$.

Lemma 15. Let $\left\{W_{j}: j \in \mathbb{Z}\right\}$ be a sequence of pairwise orthogonal closed subspaces of $L^{2}(K)$ such that $L^{2}(K)=$ $\bigoplus_{j \in \mathbb{Z}} W_{j}$. Then, for every $f \in L^{2}(K)$, there exist $f_{j} \in W_{j}$, $j \in \mathbb{Z}$, such that $f(x)=\sum_{j \in \mathbb{Z}} f_{j}(x)$. Furthermore,

$$
\|f\|_{2}^{2}=\sum_{j \in \mathbb{Z}}\left\|f_{j}\right\|_{2}^{2} .
$$

Proof. For any arbitrary function $f \in L^{2}(K)$, we have

$$
\lim _{n \rightarrow \infty}\left\|f-\sum_{j=-n}^{n} f_{j}\right\|_{2}=0,
$$

where $f_{j} \in W_{j}$, for each $j \in \mathbb{Z}$. Moreover, for a fixed $n \in \mathbb{N}$, we have

$$
\left\|\sum_{j=-n}^{n} f_{j}\right\|_{2}^{2}=\sum_{j=-n}^{n}\left\|f_{j}\right\|_{2}^{2} .
$$

Since the norm $\|\cdot\|_{2}$ is continuous, therefore the desired result is obtained by taking $n \rightarrow \infty$ on both sides of the above equality.

Theorem 16. Let $\varphi$ be the scaling function for a frame multiresolution analysis $\left\{V_{j}: j \in \mathbb{Z}\right\}$ and suppose that $W_{j}$ is the orthogonal complement of $V_{j}$ in $V_{j+1}$. Let $\Psi=\left\{\psi_{1}\right.$, $\left.\psi_{2}, \ldots, \psi_{L}\right\} \subset W_{0}$. Then, the collection

$$
\begin{gathered}
\mathscr{F}_{\Psi}=\left\{\psi_{j, k}^{\ell}(x):=q^{j / 2} \psi^{\ell}\left(\mathfrak{p}^{-j} x-u(k)\right),\right. \\
\left.j \in \mathbb{Z}, k \in \mathbb{N}_{0}, \ell=1, \ldots, L\right\}
\end{gathered}
$$

constitutes a wavelet frame for $L^{2}(K)$ with frame bounds $A$ and $B$ if and only if

$$
\left\{\tau_{k} \psi^{\ell}: k \in \mathbb{N}_{0}, \ell=1, \ldots, L\right\}
$$

forms a frame for $W_{0}$ with frame bounds $A$ and $B$.
Proof. Suppose that the system $\mathscr{F}_{\Psi}$ given by (57) is a wavelet frame for $L^{2}(K)$ with bounds $A$ and $B$. Then, it follows from (39) that the family of functions $\psi_{j, k}^{\ell}$ lies in $W_{j}$, for $\ell=$ $1, \ldots, L, j \in \mathbb{Z}$, and $k \in \mathbb{N}_{0}$.

By applying Theorem 13 to an arbitrary function $f \in W_{0}$, we have

$$
\sum_{j \in \mathbb{Z}} \sum_{k \in \mathbb{N}_{0}}\left|\left\langle f, \psi_{j, k}^{\ell}\right\rangle\right|^{2}=\sum_{k \in \mathbb{N}_{0}}\left|\left\langle f, \tau_{k} \psi^{\ell}\right\rangle\right|^{2} .
$$

Using the frame property of the system $\mathscr{F}_{\Psi}$, we have

$$
A\|f\|_{2}^{2} \leq \sum_{\ell=1}^{L} \sum_{k \in \mathbb{N}_{0}}\left|\left\langle f, \tau_{k} \psi^{\ell}\right\rangle\right|^{2} \leq B\|f\|_{2}^{2},
$$

and it follows that the collection $\left\{\tau_{k} \psi^{\ell}: k \in \mathbb{N}_{0}, \ell=1, \ldots, L\right\}$ is a frame for $W_{0}$.

Conversely, suppose that the collection $\left\{\tau_{k} \psi^{\ell}: k \in\right.$ $\left.\mathbb{N}_{0}, \ell=1, \ldots, L\right\}$ is a frame for $W_{0}$ with bounds $A$ and $B$. For any fixed $j \in \mathbb{Z}$ and $f \in W_{j}$, we have from (39) that $f\left(\mathfrak{p}^{j}.\right) \in W_{0}$. Moreover, by making use of the fact that

$$
\begin{aligned}
& \left\langle f, \psi_{j, k}^{\ell}\right\rangle=q^{j / 2} \int_{K} f(x) \overline{\psi^{\ell}\left(\mathfrak{p}^{-j} x-u(k)\right)} d x, \\
& \left\|q^{-j / 2} f\left(\mathfrak{p}^{j} \cdot\right)\right\|_{2}^{2}=q^{-j} \int_{K}\left|f\left(\mathfrak{p}^{j} x\right)\right|^{2} d x=\|f\|_{2}^{2},
\end{aligned}
$$

we have

$$
A\left\|q^{-j / 2} f\left(\mathfrak{p}^{j} \cdot\right)\right\|_{2}^{2} \leq \sum_{\ell=1}^{L} \sum_{k \in \mathbb{N}_{0}}\left|\left\langle f, \psi_{j, k}^{\ell}\right\rangle\right|^{2} \leq B\left\|q^{-j / 2} f\left(\mathfrak{p}^{j} \cdot\right)\right\|_{2}^{2} .
$$

Thus, for a given $j \in \mathbb{Z}$, the collection $\left\{\psi_{j, k}^{\ell}: k \in \mathbb{N}_{0}, \ell=\right.$ $1, \ldots, L\}$ constitutes a frame for $W_{j}$ with frame bounds $A$ and $B$.

Let $f$ be an arbitrary function in $L^{2}(K)$; then, by Theorem 13 and Lemma 15 , there exist $f_{j} \in W_{j}$ such that

$$
f=\sum_{j \in \mathbb{Z}} f_{j}, \quad\left\langle f_{i}, \psi_{j, k}^{\ell}\right\rangle=0, \quad i \neq j .
$$

Therefore, we have

$$
\begin{aligned}
\sum_{\ell=1}^{L} \sum_{j \in \mathbb{Z}} \sum_{k \in \mathbb{N}_{0}}\left|\left\langle f, \psi_{j, k}^{\ell}\right\rangle\right|^{2} & =\sum_{\ell=1}^{L} \sum_{j \in \mathbb{Z}} \sum_{k \in \mathbb{N}_{0}}\left|\sum_{i \in \mathbb{Z}}\left\langle f_{i}, \psi_{j, k}^{\ell}\right\rangle\right|^{2} \\
& =\sum_{\ell=1}^{L} \sum_{j \in \mathbb{Z}} \sum_{k \in \mathbb{N}_{0}}\left|\left\langle f_{j}, \psi_{j, k}^{\ell}\right\rangle\right|^{2} .
\end{aligned}
$$

Using (62), we obtain

$$
A \sum_{j \in \mathbb{Z}}\left\|f_{j}\right\|_{2}^{2} \leq \sum_{\ell=1}^{L} \sum_{j \in \mathbb{Z}} \sum_{k \in \mathbb{N}_{0}}\left|\left\langle f_{j}, \psi_{j, k}^{\ell}\right\rangle\right|^{2} \leq B \sum_{j \in \mathbb{Z}}\left\|f_{j}\right\|_{2}^{2} .
$$

Combining (64), (65), and Lemma 15, we have

$$
A\left\|f_{j}\right\|_{2}^{2} \leq \sum_{\ell=1}^{L} \sum_{j \in \mathbb{Z}} \sum_{k \in \mathbb{N}_{0}}\left|\left\langle f_{j}, \psi_{j, k}^{\ell}\right\rangle\right|^{2} \leq B\left\|f_{j}\right\|_{2}^{2} .
$$

This completes the proof of the theorem. 


\section{Conflict of Interests}

The author declares that there is no conflict of interests regarding the publication of this paper.

\section{References}

[1] L. Debnath and F. A. Shah, Wavelet Transforms and Their Applications, Birkhäuser, New York, NY, USA, 2015.

[2] S. G. Mallat, "Multiresolution approximations and wavelet orthonormal bases of $L^{2}(\mathbb{R})$," Transactions of the American Mathematical Society, vol. 315, no. 1, pp. 69-87, 1989.

[3] W. C. Lang, "Orthogonal wavelets on the Cantor dyadic group," SIAM Journal on Mathematical Analysis, vol. 27, no. 1, pp. 305312, 1996.

[4] Y. A. Farkov, "Orthogonal wavelets with compact support on locally compact Abelian groups," Izvestiya: Mathematics, vol. 69, no. 3, article 623, 2005.

[5] A. Y. Khrennikov, V. M. Shelkovich, and M. Skopina, "p-adic refinable functions and MRA-based wavelets," Journal of Approximation Theory, vol. 161, no. 1, pp. 226-238, 2009.

[6] S. F. Lukomskii, "Multiresolution analysis on product of zerodimensional Abelian groups," Journal of Mathematical Analysis and Applications, vol. 385, no. 2, pp. 1162-1178, 2012.

[7] S. F. Lukomskii, "Step refinable functions and orthogonal MRA on Vilenkin groups," The Journal of Fourier Analysis and Applications, vol. 20, no. 1, pp. 42-65, 2014.

[8] J. J. Benedetto and R. L. Benedetto, "A wavelet theory for local fields and related groups," The Journal of Geometric Analysis, vol. 14, no. 3, pp. 423-456, 2004.

[9] H. Jiang, D. Li, and N. Jin, "Multiresolution analysis on local fields," Journal of Mathematical Analysis and Applications, vol. 294, no. 2, pp. 523-532, 2004.

[10] F. A. Shah and L. Debnath, "Tight wavelet frames on local fields," Analysis, vol. 33, no. 3, pp. 293-307, 2013.

[11] B. Behera and Q. Jahan, "Characterization of wavelets and MRA wavelets on local fields of positive characteristic," Collectanea Mathematica, vol. 66, no. 1, pp. 33-53, 2015.

[12] F. A. Shah and Abdullah, "Nonuniform multiresolution analysis on local fields of positive characteristic," Complex Analysis and Operator Theory, 2014.

[13] F. A. Shah and Abdullah, "Wave packet frames on local fields of positive characteristic," Applied Mathematics and Computation, vol. 249, pp. 133-141, 2014.

[14] F. A. Shah and Abdullah, "A characterization of tight wavelet frames on local fields of positive characteristic," Journal of Contemporary Mathematical Analysis, vol. 49, no. 6, pp. 251-259, 2014.

[15] J. J. Benedetto and S. Li, "The theory of multiresolution analysis frames and applications to filter banks," Applied and Computational Harmonic Analysis: Time-Frequency and Time-Scale Analysis, Wavelets, Numerical Algorithms, and Applications, vol. 5, no. 4, pp. 389-427, 1998.

[16] J. J. Benedetto and O. M. Treiber, "Wavelet frames: multiresolution analysis and extensionprinciple," in Wavelet Transforms and Time-Frequency Signal Analysis, L. Debnath, Ed., pp. 3-36, Birkhäuser, Boston, Mass, USA, 2000.

[17] H. O. Kim and J. K. Lim, "On frame wavelets associated with frame multiresolution analysis," Applied and Computational Harmonic Analysis: Time-Frequency and Time-Scale Analysis,
Wavelets, Numerical Algorithms, and Applications, vol. 10, no. 1, pp. 61-70, 2001.

[18] X. Yu, "Semiorthogonal multiresolution analysis frames in higher dimensions," Acta Applicandae Mathematicae, vol. 111, no. 3, pp. 257-286, 2010.

[19] D. Ramakrishnan and R. J. Valenza, Fourier Analysis on Number Fields, vol. 186 of Graduate Texts in Mathematics, Springer, New York, NY, USA, 1999.

[20] M. H. Taibleson, Fourier Analysis on Local Fields, Princeton University Press, Princeton, NJ, USA, 1975.

[21] A. Ron and Z. Shen, "Frames and stable bases for shift-invariant subspaces of $L^{2}\left(\mathbb{R}^{d}\right)$," Canadian Journal of Mathematics, vol. 47, no. 5, pp. 1051-1094, 1995.

[22] A. Ron and Z. Shen, "Affine systems in $L^{2}\left(\mathbb{R}^{d}\right)$ : the analysis of the analysis operator," Journal of Functional Analysis, vol. 148, no. 2, pp. 408-447, 1997.

[23] B. Behera and Q. Jahan, "Multiresolution analysis on local fields and characterization of scaling functions," Advances in Pure and Applied Mathematics, vol. 3, no. 2, pp. 181-202, 2012. 


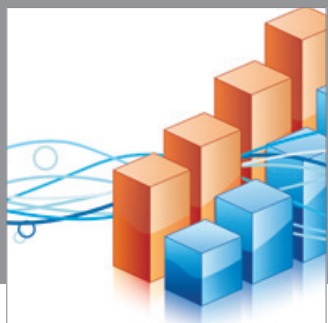

Advances in

Operations Research

mansans

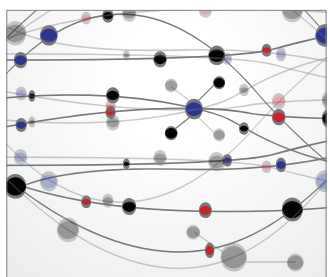

The Scientific World Journal
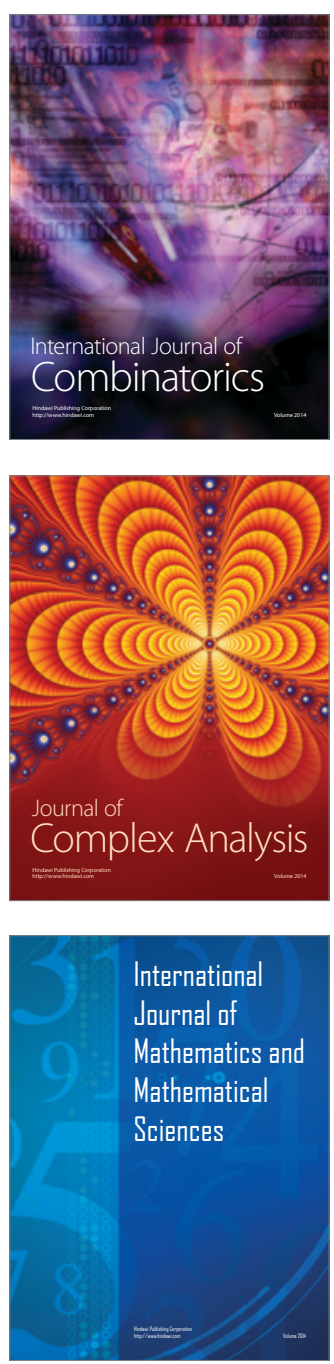
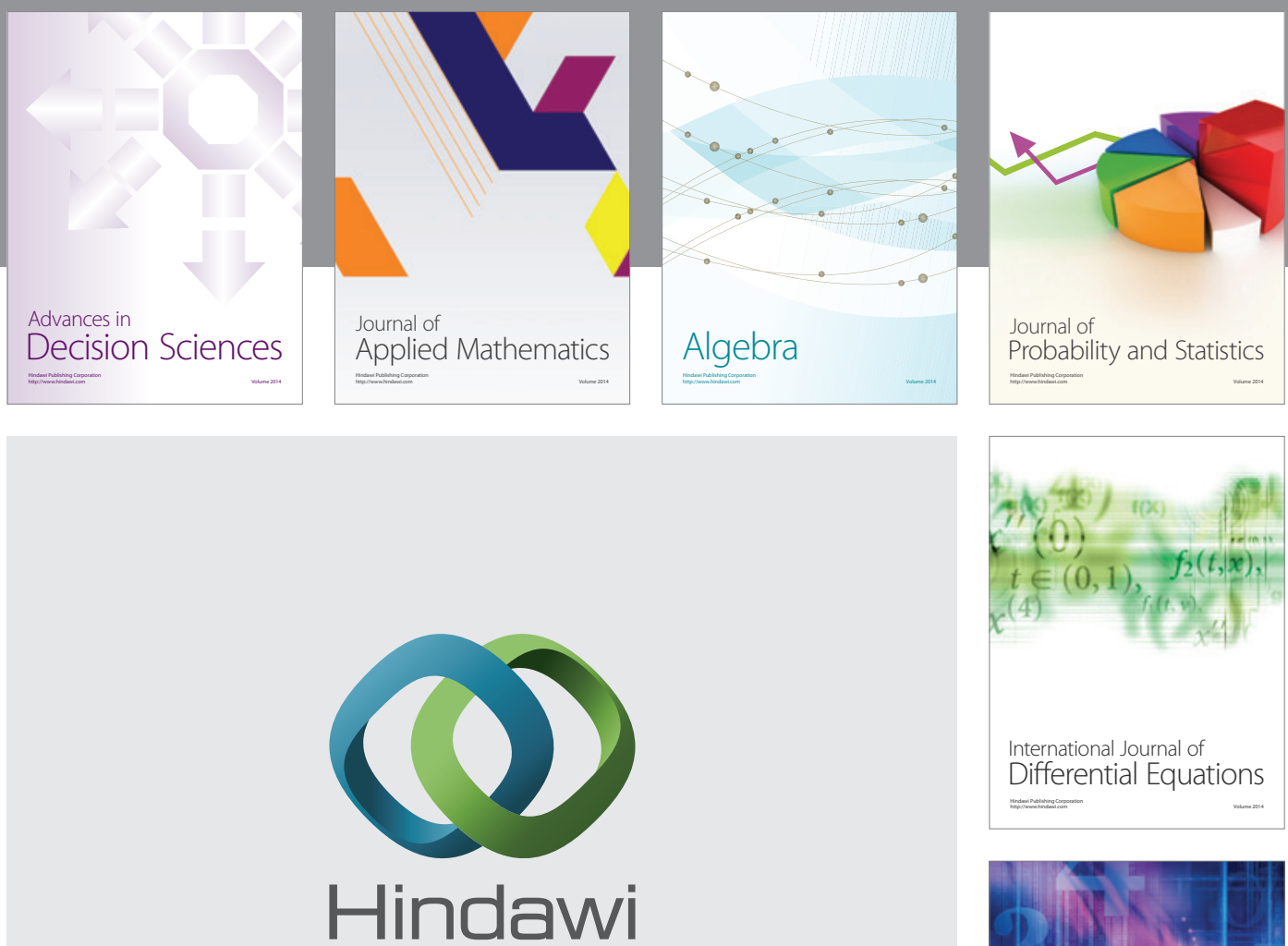

Submit your manuscripts at http://www.hindawi.com
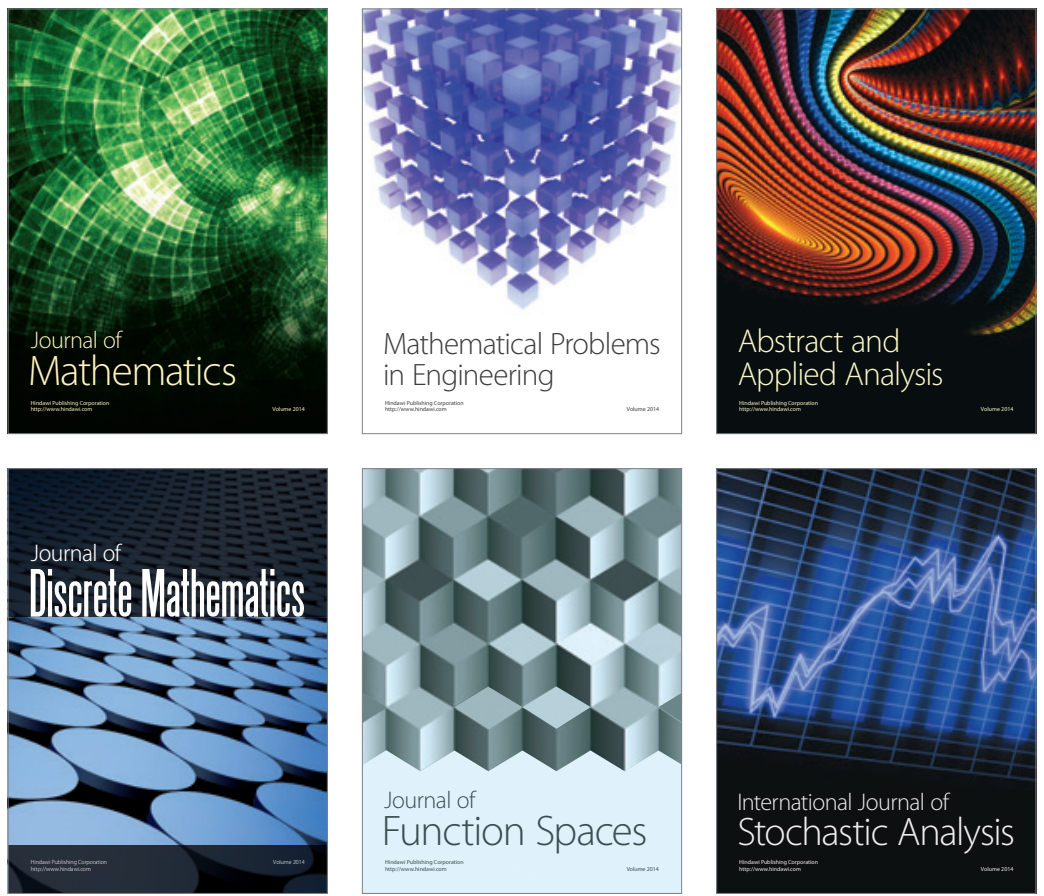

Journal of

Function Spaces

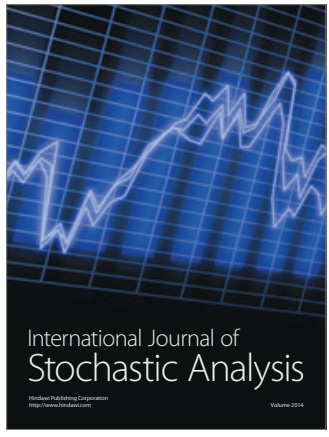

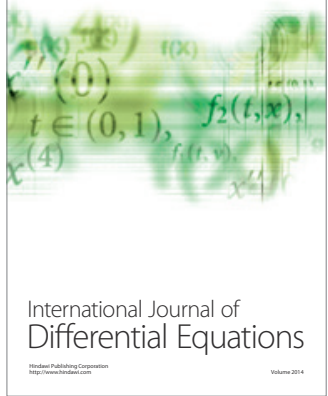
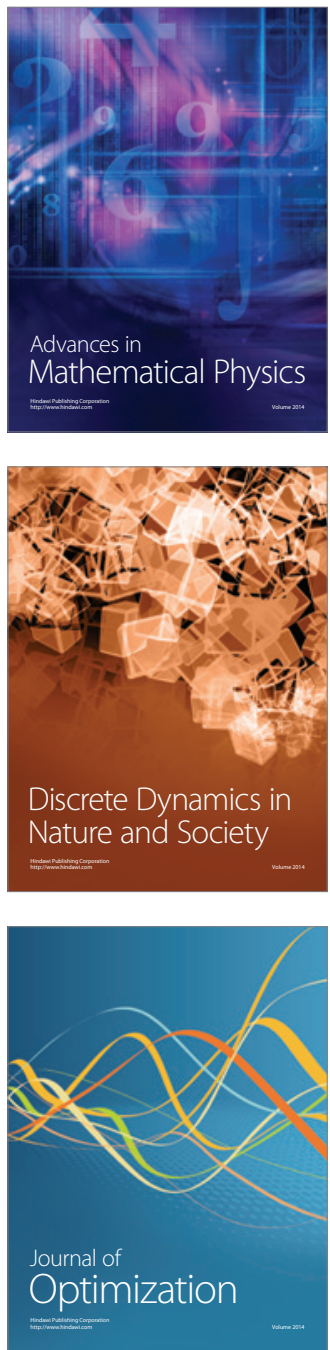